\title{
ANALYSIS OF SURFACE POST-PROCESSING TECHNIQUES FOR IMPROVEMENT OF ADDITIVE MANUFACTURED PARTS IN AEROSPACE
}

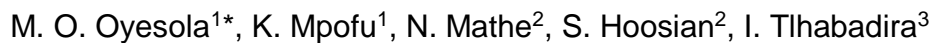 \\ ${ }^{1}$ Tshwane University of Technology, Department of Industrial Engineering, Pretoria, South Africa \\ ${ }^{2}$ Council for Scientific and Industrial Research (CSIR), Laser Enabled Manufacturing Research, National Laser \\ Centre, Pretoria, South Africa \\ ${ }^{3}$ Tshwane University of Technology, Institute for Advanced Tooling (IAT), Pretoria, South Africa \\ ${ }^{*}$ Corresponding author; e-mail: oyesolamo@tut.ac.za
}

\begin{abstract}
Additive manufacturing (AM) is a fast growing innovative technology with attractiveness to transform the manufacturing segment of the aerospace industries due to its ability to produce final usable parts. However, the surface finish of AM produced parts usually fall short of desires when compared to the conventional manufacturing method. Therefore, post-processing is often required for surface finishing as applicable in the aerospace. In this context, the AM post-processing techniques are presented along with application on parts fabricated with special attention to Ti6Al4V of titanium alloy material that is well recognised for manufacturing of aero-based parts. Machining is a popular post-processing method for finalising the surface finishing of a given part. However, machining for Ti6Al4V AM parts are known for certain challenges during process due to its inherent material properties and inadequacy for internal complex geometry parts. In this study, a series of other surface finishing techniques were investigated through testing and evaluation. The effectiveness of each processing technique is evaluated with respect to the surface topography in terms of $R a$ (arithmetical mean deviation of the profile) reduction value.
\end{abstract}

\section{Keywords:}

Additive manufacturing; Post-processing; Aerospace

\section{INTRODUCTION}

Additive Manufacturing (AM) is becoming a cutting-edge for both academia and industrial expertise as the technology transforms from unripe process stage to a suitable production process. It is simply a process that creates physical objects by adding material layer upon layer or by melting materials together [Gibson 2010]. AM technology stand the chance to revolutionise the arena of manufacturing by allowing the production of ever complex products and creation of competitive advantages as it is a digitally inclined process. The technology is progressively gaining relevance as a viable manufacturing process for the industry such as in aerospace [Uriondo 2015].

In metal AM production, few parts meet specifications "as built" due to parts usually fused and sticks inordinately to the build plate, this warrants removal efforts, hence the demand for post processing of those parts before use. Most manufacturer already have machining systems on hand, but registering parts and establishing datums for machining can be tricky especially for complex, organically shaped parts made with AM [Gibson 2010]. Therefore, accessing internal channels for geometrical accuracy or surface finishing via machining can lead to increase process cost and time. Hence, the need to examine other surface finishing treatment methods, otherwise the use of AM as a viable alternative to traditional manufacturing processes may end up being negated completely.
The techniques mostly investigated to improve the surface finishing of AM products involves the following, depending on application; (Baufeld et al 2010)

- Computer numerical control (CNC) machining

- Laser polishing

- Chemical treatments

- $\quad$ Electro polishing

- $\quad$ Slurry polishing

- Grit blasting

- Vibratory polishing

- Centrifugal barrel system

The demand for production of end usable part is increasing, and aircraft structures, parts and engines require high quality surface treatment solutions that will ensure a safe and reliable airline operation [Uriondo 2015]. Near net shaping methods are very difficult for most of the aerospace components due to complex physical morphology, and high tolerance and superior surface finish requirements [Gibson 2010]. Surface roughness in AM is highly variable and it is a dependent of location in the part (areas having the highest roughness), the process parameters [Safdar 2012] and the raw material characteristics [Frazier 2014]. Aerospace components are generally made by removing relative amounts of swarf (chips) via machining, however for material powder which can change over multiple builds as material is recycled, a feature of all materials manufactured by AM especially Titanium alloys such as Ti-6Al-4V can pose difficulties [Spierings 2011]. 


\subsection{Machining challenges with respect to AM aero based parts}

The titanium alloys are very classic materials and widely used across spectrum of industries especially in aerospace due to its array of unique properties. Ti- $6 \mathrm{Al}-4 \mathrm{~V}$ is a titanium alloy designed for good balance of characteristics, including: strength, ductility, fracture toughness, high temperature strength, creep characteristics, weldability, workability, and thermal process ability [Boyer 1996]. This alloy is therefore used for many airframe and engine parts in the aerospace application for general structural materials such as bolts, seat rails, seat buckles and the likes [Spierings 2011]. However, investigations into machining of titanium alloy (Ti6Al4V) indicates the limited applicability of machining because of its acute reactivity and low thermal conductivity response. In addition, there is usually the excessive shear localisation and strain hardening due to phase transformation of the titanium structure, and also machinability of Ti6Al4V takes place at critical cutting speed [Spierings 2011].

The popular metal AM production process for titanium alloys examined is known as powder bed fusion (PBF), further categorised into; (1) Selective Laser Melting (SLM) and Electron Beam Melting (EBM). The system uses laser or electron beam power for the distribution of powder and fuses to form metallic objects [Gibson 2010]. An illustrative diagram that depict the PBF concept is shown in Figure 1.

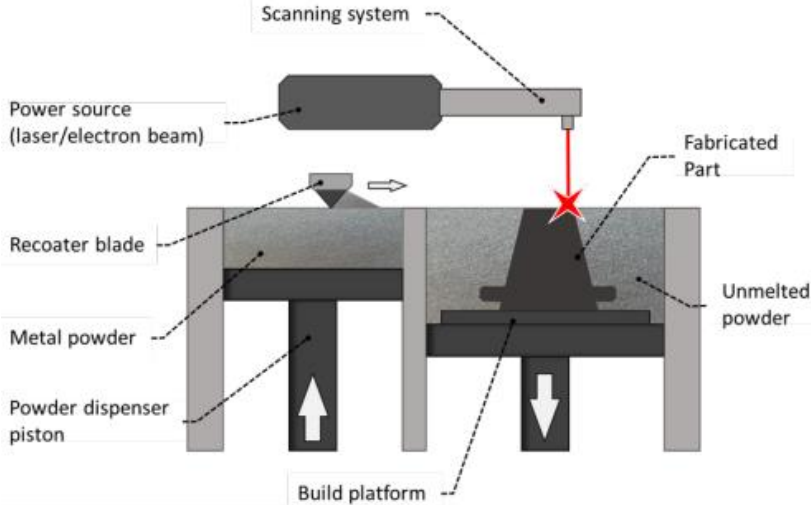

Fig. 1: The powder bed fusion process schematics [Gibson 2010].

The operation of a PBF machine is governed by the details of the scan strategy and processing parameters, depending on the process material, most build substrate may stick to the parts and recovering the parts from "as-built" state to a process defect free state often request the post processing measure. The data in Table 1 shows the mechanical properties of a typical Ti6Al4V manufactured using the PBF system (Baufeld et al 2010).
The measure of machining for post processing is considered imbalanced due to possibilities of build-up on the tool edge, low thermal conductivity leading to high temperature during machining, and a tendency for strain hardening in the machining area. This specificity of machining is established by high force and torque values, as well as extremely high thermal loads of cutting edges, which results in intensive wear and deformation of components [Frazier 2014].

The remaining section in this study discusses the experimental investigation in section 2 , using a systematic approach methodology for testing by analysing surface finishing techniques and the corresponding results in section 3 . In section 4 is the result discussion before the conclusion.

\section{INVESTIGATION AND EXPERIMENTAL SETUP}

An experimental work package was launched in a built Aeroswift chamber used for metal-additive production of part's size of $2000 \times 600 \times 600 \mathrm{~mm}$ and it uses laser melting medium for titanium powder specifically for the commercial aerospace manufacturing sector (South Africa). The system has the ability to produce geometrically complex parts to meet specification as well as minimising material wastage while processing difficult-to-machine materials. The machine has been used to achieve proof-of-concept trials and large manufacturing production capability. However, the frequency of the system's obtainable products are still of the near-net-shape characteristics that requires a further post-processing such as machining. Figure 2 shows the aeroswift production system and its chamber.

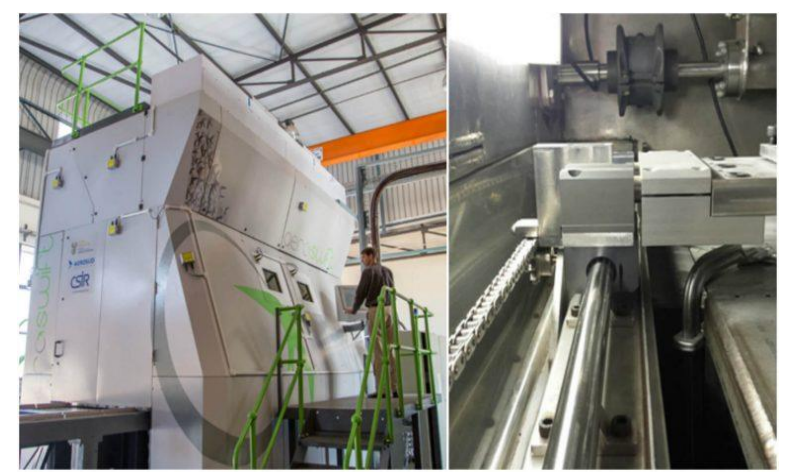

Fig. 2: The Aeroswift powder bed fusion system of advance laser additive manufacturing.

Tab. 1: The summary report of PBF production for Ti6Al4V via SLM and EBM (Data from Baufeld et al. 2010).

\begin{tabular}{lllll}
\hline Process Route & Condition & $\begin{array}{l}\text { Yield Strength } \\
(\mathbf{M P a})\end{array}$ & a UTS (MPa) & Elongation (\%) \\
\hline \multirow{3}{*}{ SLM } & As built & $736-1166$ & $1035-1407$ & $1.6-12$ \\
& Heat treated & $862-1145$ & $936-1228$ & $7-12$ \\
& b HIP & $835-973$ & $910-1088$ & $5-19$ \\
EBM & As built & $782-1150$ & $775-1237$ & $2.3-17$ \\
& Heat treated & 869 & 965 & 6 \\
& b HIP & $795-876$ & $870-978$ & $13-20$ \\
\hline
\end{tabular}

a Ultimate tensile strength; ${ }^{\mathrm{b}}$ Hot isostatic pressing 


\subsection{Component Production}

Production process assessment is needed to avoid issues relating to quality as in metal AM. This could be achieved through trial and error because every geometry alters the variables of the machine. This at times force machine operators to produce the same part several times in order to finally overcome issues such as warpage, cracking and porosity. Once successfully completed, the components have to be tested to ensure they meet the proper standards. Major relevant standards are JIS H 4600 (TAP 6400H) and ASTM G5. The yield strength of annealed materials is 825 $\mathrm{MPa}$ or higher, tensile strength is $895 \mathrm{MPa}$ or higher, and elongation is $10 \%$ or higher at room temperature used from the literature [Alfieri et al. 2017]. Figure 3 shows a Ti6Al4V titanium alloy brackets produced through PBF before surface treatment process.

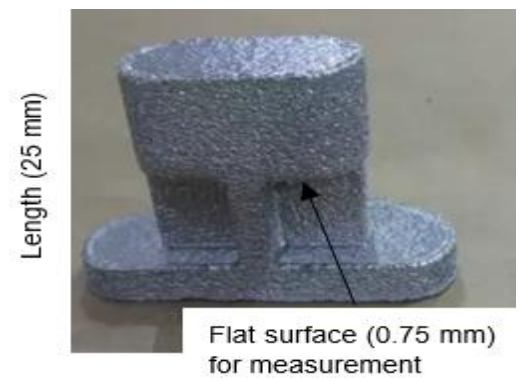

Fig. 3: Aero-based bracket component additively manufactured.

The samples of PBF product in Figure 3 above is approximately $25 \mathrm{~mm}$ in length and $17 / 25 \mathrm{~mm}$ in diameter with flat surface $0.75 \mathrm{~mm}$ for measurements. The roughness was evaluated using a Marsurf PS 1 mechanical stylus profilometer system on the flat area of the sample for Ti6Al4V with chemical composition of $\mathrm{Al}, 6.4 \%$;, $4.2 \%$; $\mathrm{Fe}$, $0.2 \% ; \mathrm{O}, 0.1 \% ; \mathrm{N}, 0.007 \% ; \mathrm{Ti}$, balance. As evaluated before and after regimen, $R a$ before treatment or as-built surface finish resulted to an average roughness $25 \mu \mathrm{m}$. The surface roughness topography as demonstrated on SEM microstructure of the built object and the built direction is as shown in Figure 4.
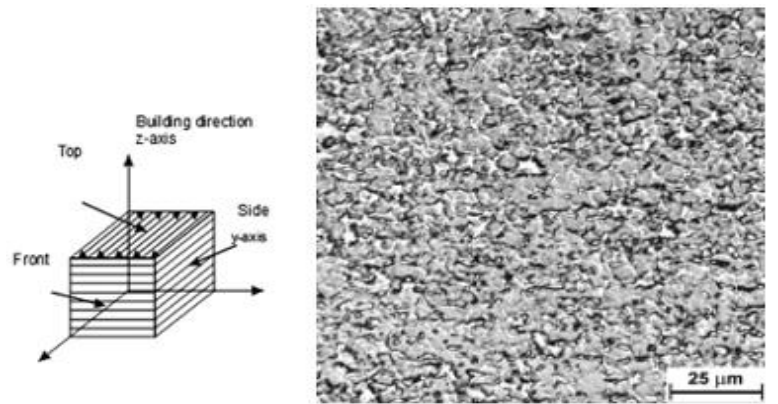

Fig 4: The SEM micrograph of as-built component.

The $R a$ result often follow this expression for a given component direction as in equation 1: [Alfieri et al. 2017]

$$
R a=\sqrt{\frac{1}{M . N} \sum_{m=1}^{M} \sum_{n=1}^{N}\left|z\left(x_{m}, \mathrm{y}_{n}\right)-\bar{z}\right|}
$$

Where $M$ and $N$ are the quantity of the measuring points $x$ and $y$ and $z$ is the distance in the $z$-direction to the middle $z$.

Typically, parts production in PBF are closer to their final shape. Notwithstanding, the surface finish is still rough with the disposed $R a$. Hence, a few experiments were conducted to obtain acceptable smooth surface finish.

\section{THE POST-PROCESSING METHODS}

In order for AM components to be considered for use in dynamic environments, the surface finish must be held in equal regard as in conventional manufacturing. Where machining is not possible on AM parts, other postprocessing methods can be investigated with respect to nature of purpose as listed and categorised in Table 2 [Perez 2004].

Tab. 2: Post processing methods with respect to nature.

\begin{tabular}{ll}
\hline Conventional & Non-conventional \\
\hline CNC machining/finishing & Electroplating \\
Vibratory bowl abrasion & Ultrasonic abrasion \\
Hot cutter machining & Chemical post-treatment \\
Optical polish & Electrochemical \\
Micro machining process & Laser micro machining \\
\hline
\end{tabular}

However, the scope for this study is based on PBF parts near-net-shape treatment listed in section 1. The explored surface finishing processes are for roughness reduction or improvement applied for Ti6Al4V as-built samples inspected and the techniques analysed in the subsections.

\subsection{Electro-polishing (EP)}

Electro-polishing (EP) is an electrochemical process that functions on the principle of reverse electroplating used to improve the surface finish of cavities that is not easily reachable using standard machining [Taylor et al. 2013]. EP technology is regarded as one of the most appropriate methods for improving the surface quality of PBF-built [Perez 2004]. The treated part is not connected as a cathode but as an anode, and when voltage is applied, the anode will be polished by the removal of surface metal particles into an electrolyte. Driven by electrical potential, the ionized particles from the treated work part will move towards the cathode enabled by the pathway provided by electrolyte [Perez 2004]. The process is the reverse of electroplating, EP is also driven by Faraday's Law, which means that the extruded features on the surface will be selectively polished more severely. Therefore in principle EP could effectively achieve selective smoothing of surface features and the improvement of surface finish.

The resulting investigation on the components explored were performed on using two-step process as attempted by a surface film, the result obtained were 1-3 $\mu \mathrm{m} R a$ improvement. However without proper solution agitation control polishing could induce pitting [Perez 2004].

\subsection{Chemical Polishing (CP)}

The chemical polishing (CP) does not employ the use of electricity as the case of electro-polishing. The processing chemical removes a thin layer of material across the entire wetted surface of the part, thereby smoothing and polishing it in the microsphere. At the same time any particles, flakes, debris, contamination and structural damage contained in the surface layer is removed. Higher removal rates along tips and edges remove the burrs to be found there up to a size of approx. $0.5 \mathrm{~mm}$ [Taylor et al. 2013]. Processing is largely independent of the form, size and structure of the parts, a simple immersion in special tanks ensures a costeffective processing of the entire surface. The main reagent used for the CP of titanium and its alloys is hydrofluoric acid (HF) that produces titanium trifluoride and hydrogen gas while polishing Ti6Al4V [Balaykin 2016]. The reaction of CP on titanium alloy is denoted in the chemical expression of 
equation 2 and by eliminating the drawbacks, nitric acid is added to HF solutions as in equation 3.

$$
\begin{aligned}
& \mathrm{Ti}+3 \mathrm{HF} \rightarrow \mathrm{TiF}_{3}+\frac{3}{2} \mathrm{H}_{2} \uparrow \\
& \mathrm{Ti}+6 \mathrm{HF}+4 \mathrm{HNO}_{3} \rightarrow \mathrm{H}_{2} \mathrm{TiF}_{6}+4 \mathrm{NO}_{2} \uparrow+4 \mathrm{H}_{2} \mathrm{O}
\end{aligned}
$$

The result for the components investigated in chemical finishing are presented in Table 3 . The samples were tested in a chemical mixture consisting $2 \% \mathrm{HF}, 20 \% \mathrm{HNO}_{3}$ and the rest water content.

Tab. 3: The results of the sample tested.

\begin{tabular}{cl}
\hline Sample $(\boldsymbol{m i n s})$ & $\mathbf{R a}(\boldsymbol{\mu m})$ \\
\hline 5 & $10-14$ \\
10 & $6-8$ \\
15 & $5-6$ \\
\hline
\end{tabular}

This polishing method is noted not suitable in precision surface finishing since it cannot remove relatively large concaves and convexes, and is more suitable in attaining glossy finishes on surface by removing fine imperfections left after pre-polishing. The process is noted as nonsatisfactory as broadly discussed [Balaykin 2016].

\subsection{Slurry Polishing (SP)}

Slurry polishing is a chemical-mechanical technique that contains more than four compositions, in which strong acids, alkalis or hazardous chemicals are normally employed. The process produces soft finish by the flow of the water acting as a lubricant to provide cleaning effect when in contact with the component under test to attain blast media. In this study, there is no experimental performance under this technique because it is a challenge to develop a kind of novel chemical mechanical polishing slurry for titanium alloy that are environmental friendly compositions [Singh 2008].

\subsection{Laser Polishing (LP)}

Laser polishing (LP) is a potential post-processing technique regularly considered to reduce the surface roughness of parts manufactured by different processes in AM. The technology is based on the concept of re-melting via laser irradiation on the material surface [Hackel 2018] The molten material flow is reallocated into cavities in order to smooth the initial topography. The final topography depends on the operating parameters of the laser on the material. The advantage is that certain critical areas where surface finish is necessary can be focused on using a laser system as compared with other techniques. LP is considered a highly efficient, very flexible, and immensely automated processing method.

In investigation components samples were tested on a surface roughness a laser ablation measuring instrument. The laser polished surfaces were analysed with profilometry, white light interferometry and light microscopy for the homogeneity of the polishing. The limitation experienced through this process is that some axes did not work. Hence, further testing were gotten for an average $R a$ of $5 \mu \mathrm{m}$ from a starting Ra of $12 \mu \mathrm{m}$. The laser system utilised for these experiments is a DML 40s manufactured by Deckel Maho Lasertec. The laser source is a Nd: YAG laser with a maximum average output power of $100 \mathrm{~W}$, and $1064 \mathrm{~nm}$ wavelength with operating frequency range of 1
$\mathrm{kHz}$ to $50 \mathrm{kHz}$. Figure 5 shows the described Deckel Maho Lasertec system for laser polishing.

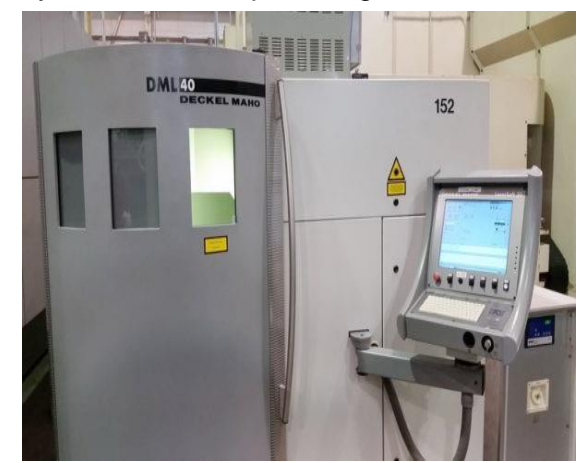

Fig. 5: Laser polishing Deckel Maho System.

\subsection{Abrasive/Grit blasting}

Abrasive blasting or Grit blasting involves the operation of forcibly propelling a stream of abrasive material against a surface under high pressure to smooth a rough surface, roughen a smooth surface, shape a surface, or remove surface contaminants [Sames et al. 2016]. Grit blasting is a stand-alone process often used for on-aircraft titanium prebond treatment, although relatively easy but requires alternate approaches for component that will be used in aggressive environments [Sames et al. 2016]. Abrasive blasting after blasting recovers the AM print media and help remove particles on inner surfaces that might otherwise not break loose until the in between the part, the operation create high levels of dust and noise however bond quality diminished with increased surface exposure time. The variety of abrasive particles with different sizes (25-90 microns) are used in this process and they are accelerated through a blasting nozzle by means of compressed air.

As investigated, an as-built handgrip was blasted for approximately 25 minutes that results in surface roughness of $15-16 \mu \mathrm{m} R \mathrm{R}$, performed at the work floor grit blaster.

\subsection{Vibratory Polishing}

The core concept of the vibratory polishing system takes the advantage of an adaptive polishing tool that conforms to the contour of the workpiece profile such that the material removal is implemented in a most efficient way [Reichelt et al. 2015]. The operation is broadly categorised into two, the round bowl and rectangular tub designs [Davidson 2005]. These systems are usually modest in size and used for the deburring and finish processing of smaller components.

The samples investigated for the process were tested in a standard media using an FSV 50 Vibrator with the as-built surface $R a 15-20 \mu \mathrm{m}$ and after vibratory polishing process of 6 hours, the Ra resulted to $0.5 \mu \mathrm{m}$. This is an indication of the process dependency.

\subsection{Centrifugal Barrel Finishing (CBF)}

The centrifugal barrel finishing (CBF) machines often provide finishing results that cannot be achieved in a standard vibratory process. A CBF typically is made up of four barrels horizontally mounted between two main drive turrets, this process requires manual loading and unloading as well as parts and media separation. Processes ranging from heavy grinding to fine polishing can be accomplished with centrifugal barrel finishing [Davidson 2005].

CBF equipment's strong suit is the ability to develop surface finishes that may require lengthy time cycles in conventional equipment and the capability to develop a 
wide range of special surface finishes required for demanding and critical applications. In this system, workpieces are fixed in a special fixture compartment and operates in circular motion through a loose abrasive or polishing media, then the spindle rotates based on speed input in clockwise or anticlockwise direction. In Figure 6 is centrifugal barrel finishing machine demonstration.

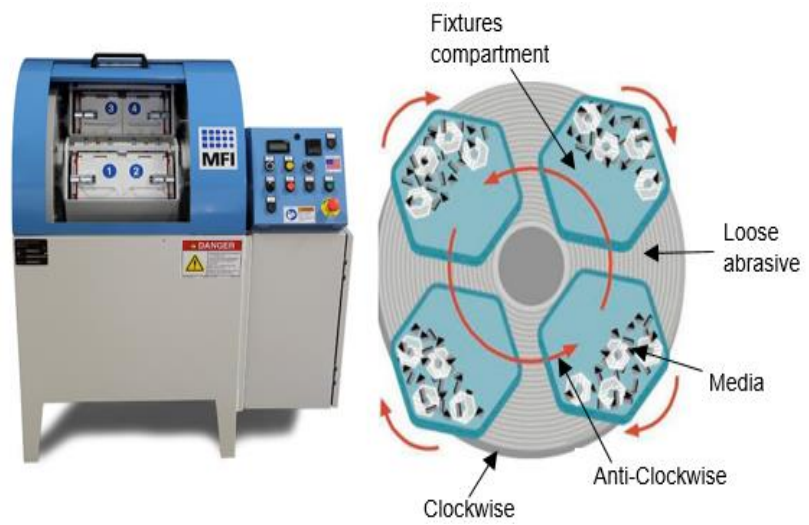

Fig. 6: The HZ-40 Centrifugal barrel finishing system.

Process evaluation were made for two samples using the $1.4 \mathrm{Cu}$.Ft./40 liter capacity. The surface roughness of $\mathrm{Ra} 27$ $\mu \mathrm{m}$ and $15 \mu \mathrm{m}$ were obtained respectively. The processing time of four hours was used to attain a finish of $<1.5 \mu \mathrm{m}$.

\section{EXPERIMENTAL RESULTS AND TECHNIQUES DISCUSSION}

Evaluation through systemic investigation offers a way to understand and improve the use of methods that are useful, feasible, proper, and accurate [Schrage 1994]. In order to have a reference, sample series were conducted for an asbuilt metallic AM components in section 2.1 which showed a high initial surface roughness $25 \mu \mathrm{m}$ prior to final finishing. The component is used primarily for loadbearing application in the aerospace. The standard guidelines for the surface qualities for aerospace applications typically call for a surface roughness of $R a=3.2 \mu \mathrm{m}$ or even lower for cast or wrought parts [Ribeiro 2003]. Clearly, the roughness provided in Table 4 reflects the exceed post processing technique for this value. A few experiments were conducted to evaluate average roughness values after roughness optimisation via EP, CP, LP, Blasting, VP and CBF concurrently. Table 4 summarised observation in roughness removal and Figure 7 shows the final obtained surface finishing of the component.
Tab 4: Summarised observation in roughness removal.

\begin{tabular}{|c|c|c|}
\hline $\begin{array}{l}\text { Treatment } \\
\text { Technique }\end{array}$ & $\begin{array}{l}\text { Ease of } \\
\text { operation }\end{array}$ & $\begin{array}{l}\text { Surface } \\
\text { reduction } \\
(\mathrm{Ra}) \mu \mathrm{m}\end{array}$ \\
\hline $\begin{array}{l}\text { Electro- } \\
\text { polishing }\end{array}$ & $\begin{array}{l}\text { Skilled } \\
\text { operator }\end{array}$ & $1-3$ \\
\hline $\begin{array}{l}\text { Chemical } \\
\text { polishing }\end{array}$ & $\begin{array}{l}\text { Easy } \\
\text { operation } \\
\text { with safety } \\
\text { concern }\end{array}$ & $4-5$ \\
\hline $\begin{array}{l}\text { Slurry } \\
\text { polishing }\end{array}$ & $\begin{array}{l}\text { Easy. Some } \\
\text { training } \\
\text { required }\end{array}$ & Not tested \\
\hline $\begin{array}{l}\text { Laser } \\
\text { polishing }\end{array}$ & $\begin{array}{l}\text { Skilled } \\
\text { operator } \\
\text { required }\end{array}$ & $<5$ \\
\hline Blasting & Easy to use & $\begin{array}{l}\text { 8-12 } \\
\text { (measured) }\end{array}$ \\
\hline $\begin{array}{l}\text { Vibratory } \\
\text { polishing }\end{array}$ & $\begin{array}{l}\text { Some } \\
\text { training } \\
\text { required }\end{array}$ & 0.4 \\
\hline $\begin{array}{l}\text { Centrifugal } \\
\text { Barrel } \\
\text { Finishing }\end{array}$ & $\begin{array}{l}\text { Fairly easy to } \\
\text { use }\end{array}$ & $<0.6$ \\
\hline
\end{tabular}
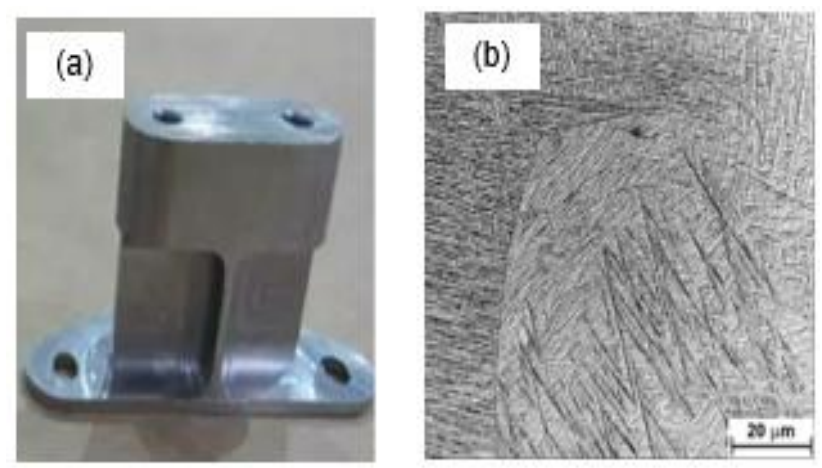

Fig. 7: (a) The bracket component final regimen and (b) the SEM micrograph view.

Out of the applied surface finishing processes, laser polishing and blasting methods exhibit best result in roughness reduction and performances. This appears to be mainly for the effect of material removal, where surface roughness furrows and irregularities were erased in a systematic graually process. This deduction was apparent both in roughness values and SEM micrographs, which reveals the smoothness surface topography. 


\section{CONCLUSION}

Additively manufactured metallic parts shows a high surface roughness and in order to reduce the roughness, different mechanical surface finishing processes were investigated. Therefore, the post-processing methods of electro-polishing, chemical polishing, slurry polishing, laser polishing, blasting methods, vibratory polishing and central barrel finishing techniques were all investigated.

The laser based process provides an alternative to the follow up abrasion and grit blast treatments which are expensive, hazardous, polluting, and less precise. The chemical-dip technique would have been preferable but this report preliminary testing of a surface preparation technique using laser polishing ablation as a replacement for the chemical etch and abrasive processes currently applied to ti-6al-4v alloy adherents. The study of various works indicates aside from machining the laser polishing is found very effective as well as the blasting technique more efficiently. In conclusion, although all surface finishing processes achieved a substantial roughness improvement, in reality, user experiences makes post-processing technique varies from resources to technology and the part being treated for specify required Ra value.

\section{ACKNOWLEDGMENTS}

The study was supported by the Department of Science and Technology (DST) and Council for Scientific and Industrial Research (CSIR), South Africa. The authors would like to acknowledge the expertise of Mr. Hoosian Shaik as well as other project partners whose input in this research made this work possible.

\section{REFERENCES}

[Gibson 2010] Gibson, I., A. Mateus, and P. Bartolo, RapidPRE (2010): A new additive manufacturing technique based on reaction injection moulding. Annals of DAAAM 2010 \& Proceedings, 21(1): p. 1589-1590.

[Uriondo 2015] Uriondo A, Esperon-Miguez $M$, Perinpanayagam $\mathrm{S}$. The present and future of additive manufacturing in the aerospace sector: A review of important aspects. Proceedings of the Institution of Mechanical Engineers, Part G: Journal of Aerospace Engineering. 2015 Sep; 229(11):2132-47.

[Safdar 2012] Safdar, A., et al., Effect of process parameters settings and thickness on surface roughness of EBM produced Ti-6Al-4V. Rapid Prototyping Journal, 2012. 18(5): p. 401-408.

[Frazier 2014] Frazier W.E., Metal Additive Manufacturing: A Review, Journal of Materials Engineering and Performance, 2014. 23: p, 1917-1928.

[Spierings 2011] Spierings, A.B., N. Herres, and G. Levy, Infuence of the particle size distribution on surface quality and mechanical properties in $A M$ steel parts. Rapid Prototyping Journal, 2011. 17(3) : p. 195-202.

[Baufeld 2010] Baufeld, Bernd, Omer Van der Biest, and Rosemary Gault. "Additive manufacturing of Ti-6Al-4V components by shaped metal deposition: microstructure and mechanical properties." Materials \& Design 31 (2010): S106-S111.
[Boyer 1996] Boyer, R.R. An overview on the use of titanium in the aerospace industry. Materials Science and Engineering A213 1996, 213, 103-114.

[Ribeiro 2003] Ribeiro, M.V.; Moreira, M.R.V.; Ferrira, J.R. Optimization of titanium alloy (Ti-6Al-4V) machining. Journal of Materials Processing Technology 2003, 143144, 458-463.

[Vittorio 2017] Vittorio Alfieri, Paolo Argenio, Fabrizia Caiazzo, and Vincenzo Sergi Reduction of Surface Roughness by Means of Laser Processing over Additive Manufacturing Metal Parts. Materials (Basel). 2017 Jan; 10(1): 30.

[Taylor 2013] Taylor, E. J., M. Inman T. Hall, S. Snyder, and A. Rowe. 2013. "Electropolishing of Niobium SRF Cavities in Low Viscosity Aqeous Electrolytes without Hydrofuouric Acid." SRF 2013, International Conference on RF Superconductivity, Paris, France

[Perez 2004] Perez, Nestor. 2004. Electrochemistry and Corrosion Science. Boston: Kluwer Academic Publishers.

[Balaykin 2016] A.V. Balaykin, E.A. Nosova, N.V. Galkina, Research of Residual Stress after Deformation and Annealing of a Titanium Alloy Ti-5Al-5Mo-5V-1Cr-1Fe Billets // IOP Conference Series: Materials Science and Engineering, Vol. 142. Issue 1. (2016).

[Singh 2008] Singh, R.; Khamba, J. Comparison of slurry effect on machining characteristics of titanium in ultrasonic drilling. Journal of Materials Processing Technology 2008. 197, 200-205.

[Hackel 2018] Lloyd Hackel, Jon R. Rankin, Alexander Rubenchik, Wayne E. King, Manyalibo Matthews, Laser peening: A tool for additive manufacturing post-processing, Additive Manufacturing, Volume 24, 2018, Pages 67-75

[Reichelt 2015] Reichelt, Sarah \& Mertens, Tobias \& Greitemeier, Daniel \& Carton, Louise \& Schoberth, Achim. (2015). Surface finishing of additive manufactured Ti-6Al$4 \mathrm{~V}$ - a comparison of electrochemical and mechanical treatments.

[Davidson 2005] Davidson, D.A. Surface finishing reaches new heights: Mass media finishing techniques can improve aircraft part performance and service life. Metal Finish. 2005, 103,25 - 28.

[Sames 2016] W. J. Sames, F. A. List, S. Pannala, R. R. Dehoff \& S. S. Babu (2016): The metallurgy and processing science of metal additive manufacturing, International Materials Reviews

[Schrage 1994] Schrage, D.P., Mavris, D.N. (1994), "Technology for Affordability - How to Define, Measure, Evaluate, and Implement It?", 50th National Forum of the American Helicopter Society, Washington, D.C., May 1113. 